\title{
DÜBLIN
}

Technological University Dublin

ARROW@TU Dublin

2006-12

\section{MEMS Mobile GIS: a Spatially Enabled Fish Habitat Management System}

\author{
Andrea Rizzini \\ University College Dublin \\ Keith Gardiner \\ Technological University Dublin, keith.gardiner@tudublin.ie \\ Michela Bertolotto \\ University College Dublin, michela.bertolotto@ucd.ie
}

See next page for additional authors

Follow this and additional works at: https://arrow.tudublin.ie/dmccon

Part of the Computer Sciences Commons, and the Environmental Sciences Commons

\section{Recommended Citation \\ Rizzini, A. et al (2006) MEMS Mobile GIS:a spatially enabled fish habitat management system. In W2GIS 2006, (eds J.D. Carswell and T. Tezuka) LNCS 4295, pp. 113-122, 2006: Hong Kong, China; December.}

This Conference Paper is brought to you for free and open access by the Digital Media Centre at ARROW@TU Dublin. It has been accepted for inclusion in Conference papers by an authorized administrator of ARROW@TU Dublin. For more information, please contact arrow.admin@tudublin.ie, aisling.coyne@tudublin.ie, gerard.connolly@tudublin.ie.

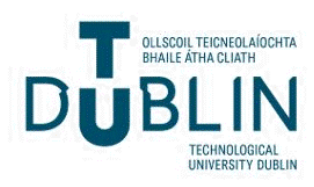




\section{Authors}

Andrea Rizzini, Keith Gardiner, Michela Bertolotto, and James Carswell

This conference paper is available at ARROW@TU Dublin: https://arrow.tudublin.ie/dmccon/86 
Dublin Institute of Technology

ARROW@DIT

Articles

Digital Media Centre

2006-12-01

\title{
MEMS Mobile GIS: a spatially enabled fish habitat management system
}

\author{
Andrea Rizzini \\ University College Dublin \\ Keith Gardiner \\ Dublin Institute of Technology, keith.gardiner@dit.ie \\ Michela Bertolotto \\ University College Dublin, michela.bertolotto@ucd.ie \\ James D. Carswell \\ Dublin Institute of Technology, jcarswell@dit.ie
}

\section{Recommended Citation}

Rizzini, Andrea:MEMS Mobile GIS:a spatially enabled fish habitat management system. In W2GIS 2006,( eds J.D. Carswell and T.

Tezuka) LNCS 4295, pp. 113-122, 2006: Hong Kong, China; December, 2006 


\title{
MEMS Mobile GIS: A Spatially Enabled Fish Habitat Management System
}

\author{
A. Rizzini ${ }^{1}$, K. Gardiner ${ }^{2}$, M. Bertolotto ${ }^{1}$, and J. Carswell ${ }^{2}$ \\ ${ }^{1}$ School of Computer Science and Informatics, University College Dublin, Belfield, \\ Dublin 4, Ireland \\ ${ }^{2}$ Digital Media Centre, Dublin Institute of Technology, Aungier Street, Dublin 2, \\ Ireland
}

\begin{abstract}
Spatially enabled computing can provide assistance to both web-based and mobile users by exploiting positional information and associated contextual knowledge. The Mobile Environmental Management System (MEMS) is a proof of concept prototype that has been developed in order to simplify administrative duties of biologists at the Department of Fisheries and Oceans (DFO), Canada. MEMS aims to deliver contextaware functionality aided by visualization, analysis and manipulation of spatial and attribute datasets. The resulting application delivers a set of functions and services that aids the DFO's biologists in making everyday management decisions.
\end{abstract}

\section{Introduction}

This paper describes the techniques used in the development of a web-based and Mobile Environmental Management System (MEMS). The current prototype has been tailored to deliver context-aware functionality aided by visualization, analysis and manipulation of spatial and attribute datasets. Context associated knowledge is an intelligent process which retrieves specific data for users. It is achieved by combining knowledge gained about data processed in the past with the activities planned by the user, together with other activity dependencies such as geographical location. The MEMS datasets are provided by the Canadian Department of Fisheries and Oceans (DFO) and the prototype is customized to the specific needs of the Great Lakes Laboratory for Fisheries and Aquatic Sciences (GLLFAS) Fish Habitat Management Group's requirements for fish species at risk assessment. Currently, biologists have only access to the fisheries data from their office. This greatly prevents them from interacting with the data in a real-time environment, reducing their productivity and effectiveness in the field. Spatially enabling a mobile device allows mobile GLLFAS biologists to make informed decision immediately. This research concerns DFO priorities specifically to administer the fish habitat provision of the Fisheries Act, in particular those that are aimed at preventing the harmful alteration, disruption or destruction of fish habitat. This is done to conserve, restore and develop the productive capacity of habitats for recreational, commercial and subsistence fisheries both in the freshwater and marine environments [1, 2]. The functionality that GLLFAS 
biologists require from the MEMS prototype includes access to geo-referenced maps and imagery, to overlay the current position on a map and to manipulate (e.g. input/edit/query) attribute data in the field while wirelessly connected (where possible) to the office database 12 . Additional functionality also required by the DFO is the ability to record, edit and view multimedia annotations, perform scientific/common-name conversion and graph generations of results. The current "fish species at risk" work-flow, whereby scientists enter textual/ pictorial information on paper field data sheets is inefficient, has potential for inaccuracies during both initial recording and subsequent data entry phases, and does not facilitate knowledge sharing between staff. Also, different types of information may be stored in different locations and valuable time can often be lost trying to correlate data in order to make decisions. The proposed MEMS system has the following advantages over current practice:

1. Facilitates knowledge sharing and data analysis/synthesis.

2. Supports effective communication between different staff at different physical locations (e.g. scientists in the lab and colleagues in the field).

3. Allows important multimedia data and associated annotations to be combined with text-based records.

4. Saves time and money by reducing paperwork and allows staff to input and access information anywhere at any time without having to return to dedicated access points.

5. Reduces error by reducing latency between collection and data entry, as well as paperwork.

\section{Related Work}

There are few contemporary systems that can be compared to the current prototype. One in particular is the Mobile Environment Monitoring System (MEMoS) 3] developed at the Multi-purpose Environmental Modelling Facility of the University of Windsor in Canada in collaboration with the ConestogaRovers \& Associates (CRA). This system is designed to deliver real-time environmental information using rugged field computers, radio technologies (CRA's OpenRTU [4]) and highly modular, interoperable software. The system also allows the user to rapidly collect and integrate spatially referenced data from a range of mobile environmental data collectors and update a GIS data warehouse in real-time making data available to a web-based client application instantly. This system adopts a distributed approach and uses a remote base station and mobile data loggers. The remote base station is a vehicle, containing the OpenRTU receiver and a computer with an Oracle Spatial database server (see Figure 1). The OpenRTU system is a remote process monitor that collects data from connected data loggers. The station allows multiple roving data collectors to wirelessly send recorded structures, where the incoming data are routed to appropriate tables in a local database. The desktop, in the base station, contains a web-enabled client application which is used to generate real-time results for the 
data collected. The mobile data loggers are backpack type equipment containing the OpenRTU transmitter connected to a series of environmental sensors and a rugged Tablet PC for data input.

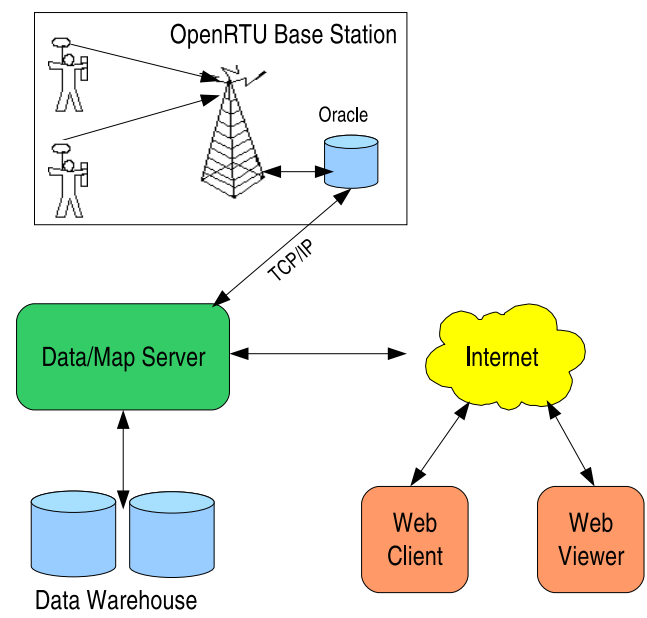

Fig. 1. MEMoS System Architecture

When the base station has finished collecting data, it connects via wired or wireless TCP-IP to the data warehouse, updating the new datasets. The advantage of this system is that it uses an inexpensive communication infrastructure between the base station and the client station. If the communication had to be established via GPRS, running cost could dramatically escalate. A possible disadvantage in running the OpenRTU transmission medium, is the actual working range. Also, the disjunctive update of the data warehouse means that information may not be accurate until such updates are completed.

\section{Technologies}

The MEMS prototype uses an Oracle Spatial database. Oracle Spatial provides a platform that supports a wide range of applications from automated mapping/facilities management and Geographic Information Systems (GIS), to wireless location services and location-enabled e-business. Oracle Spatial is integrated into the extensible Object Relational Database Management System (ORDBMS), which allows access to the full functionality and security of the underlying DBMS 7]. Along with the database, the Oracle proprietary OC4J application server is used. The application server is a component of Oracle, and is installed automatically. The application server acts as deployment platform for Oracle applications. The Oracle Enterprise Manager application is installed in the OC4J as a web application where the user can manage the database. In addition MEMS uses eSpatial Solutions [8] iSmart Suite to consolidate all forms of 
spatial data. The suite is based on a J2EE application development environment where the user can easily integrate spatial components. iSmart is a collection of tools that enables developers to build and deploy spatial applications using a set of standard procedures. These tools offer developers a high-level development environment which is several time faster than developing the application from a base Java Development Environment.

\section{System Architecture}

The MEMS prototype uses a typical three-tier architecture [5] (see Figure 2) for enterprise information systems, composed of the client layer, application server layer, and the database layer. This architecture focuses on the development of services for a versatile, extendible (J2EE) application, instead of giving GIS capabilities to a large monolithic application. The implementation of the application follows a modularization approach. Functionality is encapsulated in packages avoiding cross functionality dependencies and hence enforcing stability. The communication between the client layer and the database are conducted

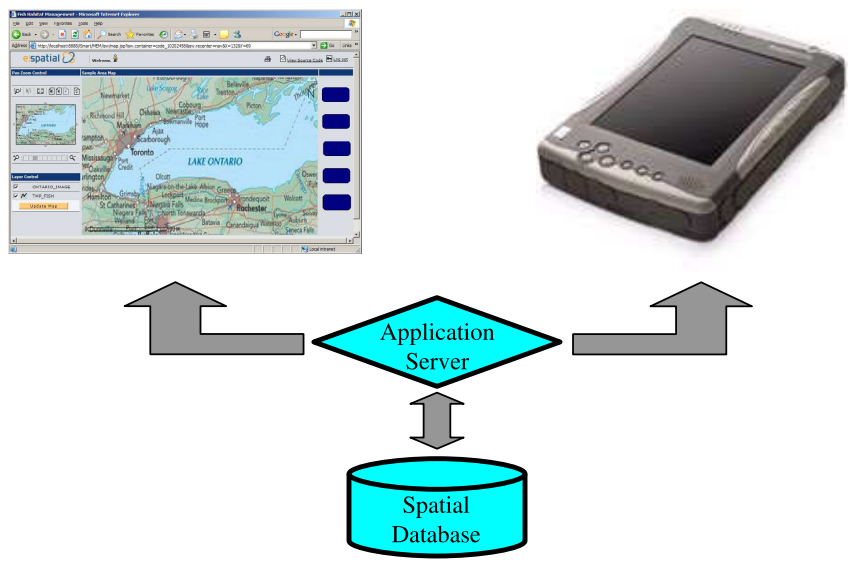

Fig. 2. MEMS System Architecture

through the application server layer. The application server layer acts as a mediator. It ensures a minimum amount of data is transferred between the server and the client. Requests from the client are interpreted and executed by the application server. A response is assembled and sent back to the client. With this type of architecture, the processing load is balanced, requiring a minimum level of computing power on a thin client[11]. The client layer consists of a comprehensive web-interface that provides biologists with the ability to input, edit, analyze and annotate data over the Internet. A Tablet PC [6] is used equipped with a Global Positioning System (GPS) receiver and a General Packet Radio Service (GPRS) network connection. As the user navigates in the field their positioning and orientation is displayed on the web-interface on a geo-referenced map. 


\section{System Functionality and GUI}

The MEMS prototype uses a comprehensive graphical user interface (GUI) to interact with the user. The GUI is divided into three main parts. The left panel (See Figure 3) is the "Navigation panel". It contains navigation functions used by the user to navigate the map (zoom to area, click $\mathrm{x} / \mathrm{y}$ coordinates, zoom to extent, back to original view, previous view, refresh map). The navigation map

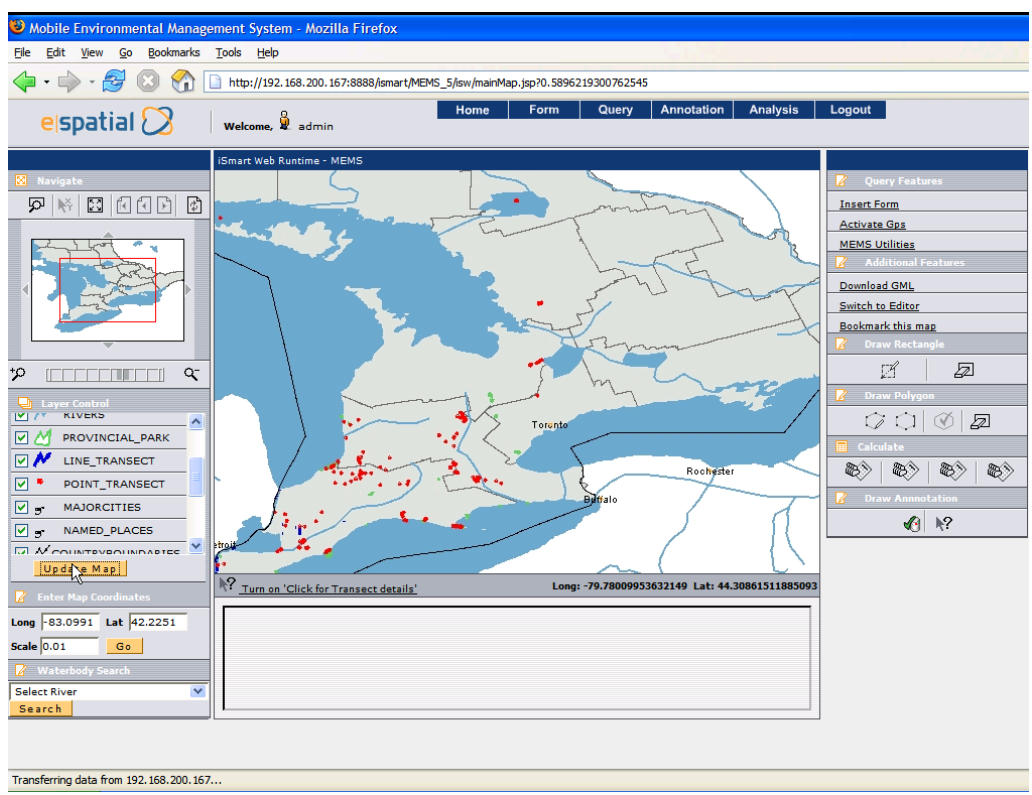

Fig. 3. MEMS Graphical User Interface

contains a red square which represents the area displayed by the main map. This is designed to help the user navigate more effectively when zoomed into a small area on a map. The "Layer Control" is located below the navigation map and is used to select layers on the main map. The last two controls on the navigation panel contain "Map Coordinates", used to centre the main map with any given coordinates and the "Search River" function control, used for searching recorded habitats. In the centre of the application (See Figure 3) the "Map Pannel" contains the main map. The main map offers "Clickable Map" functionality that enables the user to select features on the map and displays information about them. The "Tools panel" contains controls for functions which are explained in the following sections.

\subsection{GPS Acquisition}

This is a core module as the distributed MEMS application forces the client to acquire GPS coordinates first and then send them to the application server. 
As the client was not designed to be installation independent it is necessary to execute the Java application remotely. One of the technologies that Java offers is JNLP (Java Network Launching Protocol). JNLP applications are launched

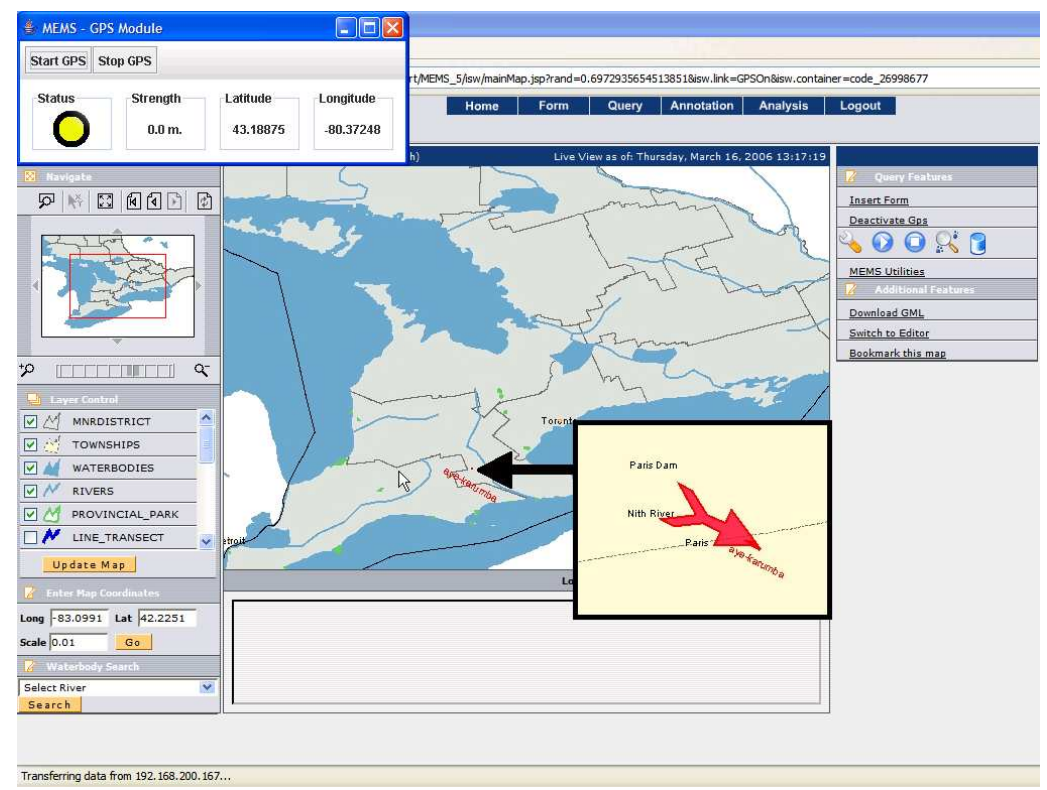

Fig. 4. Gps Module

using Java Web Start as part of the Java Runtime Environment. The corresponding GPS application registers each client IP address and host-name with the application server so that multiple feeds can be displayed on the map. The screenshot (See Figure 4) shows the GPS acquisition overlayed on the MEMS map. In the top left corner the GPS module control is shown and is launched by the java Web Start while in the center the black square is a zoomed image of the GPS feed.

\subsection{Multimedia Annotation}

Multimedia annotation is an advanced feature of MEMS. Annotation is a simple way to record data on the fly. For example, a new species of fish could be encountered and visual evidence would be of great assistance. This functionality enables the user to embed video, audio, text and image annotations on the map. These annotations are uploaded to the database as BLOB data along their associated coordinates. Using JSP and Servlet technology, the user is required to enter some text describing the annotation. If the annotations are video, audio or image annotations a file is also required to be uploaded. The file byte-stream is transferred to the application server, along the text and the current location, where it is inserted in the database. When this procedure is completed the map 


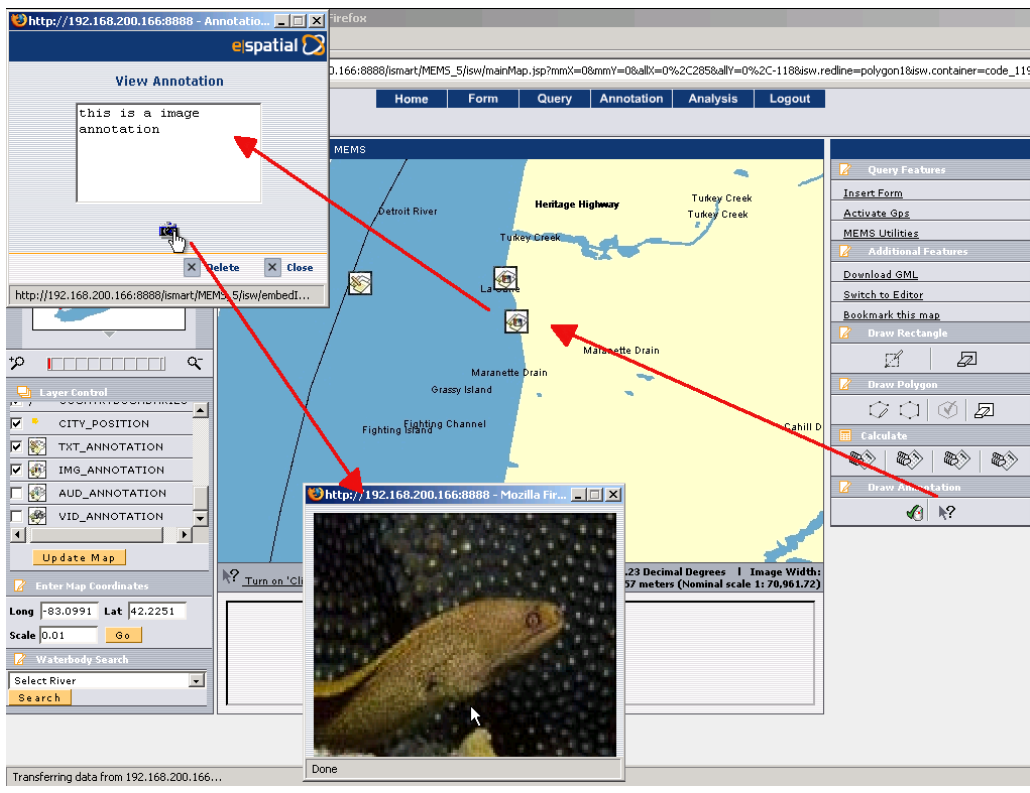

Fig. 5. Multimedia Annotation

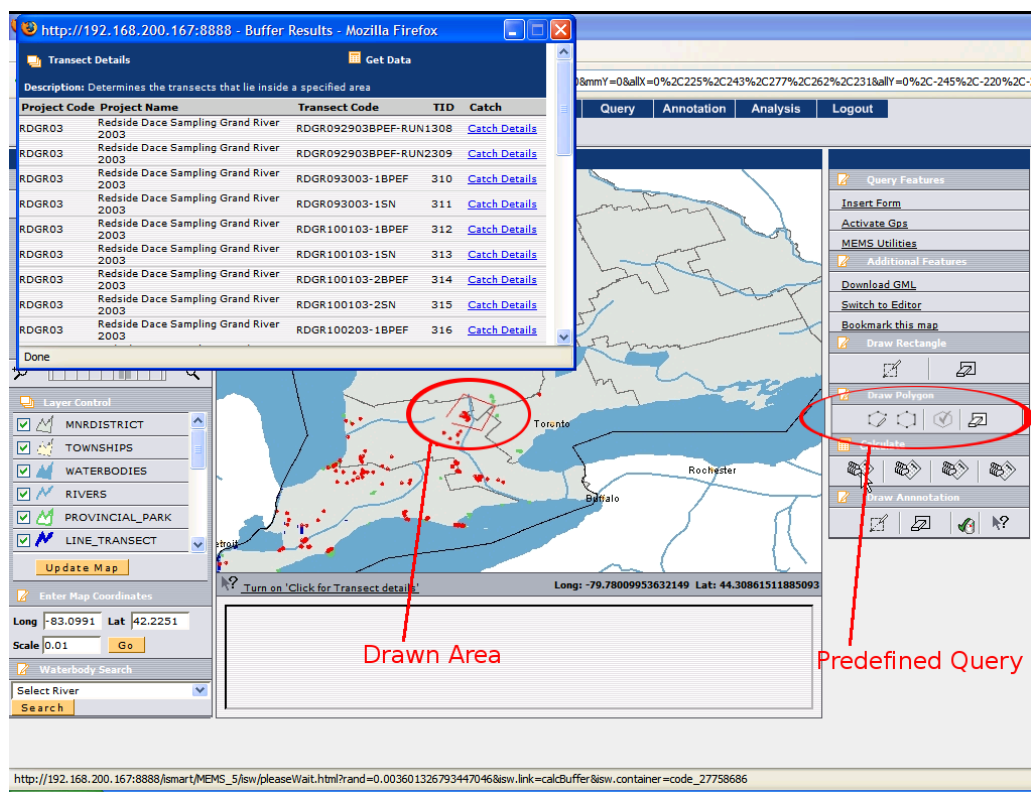

Fig. 6. Spatial Queries 
is refreshed and an icon representing the annotation is displayed. (See Figure 5). The user can view the annotations by clicking on a corresponding icon. This action opens a pop-up window that displays any text associated with the annotation and a link to any multimedia data.

\subsection{Spatial Queries}

The Spatial Queries Tool is an advanced tool which enables biologists to quickly and easily query the database. Spatial Queries are complex in nature and require a great deal in understanding of Oracle Spatial and the Sequel Query Language (SQL). Therefore, an easy to uses interface was developed to enable biologists to perform them. The tool is very easy to use. The biologists are only required to draw an area on the map and it is then possible to execute a number of predefined spatial queries on the selected area. The polygon highlighted on the map (See Figure 7)is the actual spatial component and the highlighted buttons are the predefined queries requested by the GLLFAS biologists. The procedure starts by drawing a polygon or a square on the map. The red dots on the map represent locations where the biologists have previously recorded. The result of any given query is parsed and displayed in a pop-up web-page. The module has been implemented using extensive JavaScript to draw and calculate the points of the polygon. Once the polygon is drawn a JSP is used to transform the polygon to an Oracle JGeometry object. This object is then stored in the user session ready for use in the query. Using iSmart, a SQL command is embedded in the application and is executed when the query button is clicked upon. The result is temporarily stored in the user session, where it will be parsed and displayed by the application.

\subsection{Offline Module}

The offline module was only considered after the first visit to the DFO. It was observed that the area the biologists were working had intermittent cellular signal. This unexpected fact meant that the development of a backup system was necessary. It was difficult to detect or predict network availability but fortunately the biologist were aware of cellular network presence in areas they need to investigate. Using this information the offline module was designed. The module is implemented using stand-alone Java and it is developed using the iSmart technology. The Offline Module also called MEMSOffline is a standalone application that connects directly to the online application server where the biologist is asked to select an area of interest. The selected area is compressed into a zip type file and saved. The MEMSOffline compressed offline file contains all the data required by the biologist and the application. This offline application enables the user to work remotely without the GPRS connection. In the field, the offline application reads in the compressed file and displays the map using the iSmart Editor application, enabling the biologists to perform spatial queries, insert new forms, look at previously recorded forms, display GPS feeds and navigate the map. It also offers restricted multimedia annotation functionality. Data which is changed or added during the sampling session is stored and saved in 


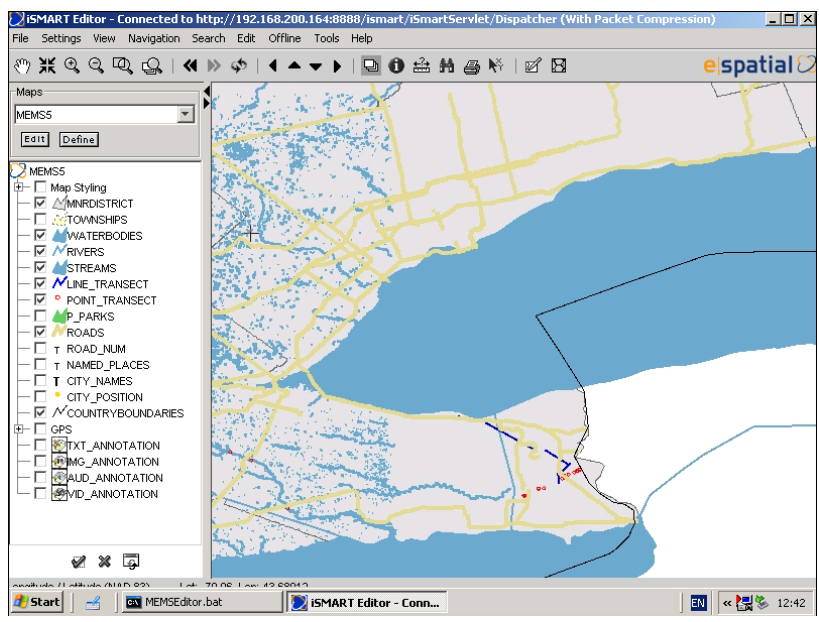

Fig. 7. MEMSOffline

the offline file. When the application establishes a network connection and can connect to the application server, the data is synchronized with the server and any additions are added to the database.

\subsection{Forms Module}

This Forms module was requested from the GLLFAS biologists and is designed accordingly. A requirement for this part is that the biologists need to navigate back and forward from different "fish forms", without committing them to the database, until all the data has been successfully collected. This behavior proved difficult to implement in a Client/Server environment. In order to achieve this AJAX (Asynchronous JavaScript and XML) technology is used. AJAX 9] uses a series of JavaScripts to compose and send a URL request to a server side page (JSP, Servlet, PHP, CGI) and return and display the response (using DOM objects) in the web-application. This is done without the need to reload the page. Using this technology, the form changes can be posted to the corresponding Java data object in the session. If the user changes a field in the form, the corresponding Java object is updated immediately. This module also offers the ability to dynamically add new "fish forms" as required. If biologists encounter a species which has not been recorded they can dynamically add another field to the current form to accommodate it. This module has also been designed to easily handle database errors or warnings that may arise during program execution. If constraints are triggered, they can easily be interpreted and displayed the user. If the unique project code has been previously entered in the database a warning message is displayed to the user. Another feature is the ability to validate the fields in the forms. Extensive JavaScripts are used to iterate through the fields of the page and check for types (Double-Integers), range and white spaces. 


\section{Conclusions}

MEMS is currently deployed at the GLLFAS headquarters, where extensive fielding testing is been carried out. The system is fully operational and it offers a spatially-enabled mobile and adaptable service for the biologists, ensuring better utilization of resources. The advantages of the system go beyond the system's functionality. This system delivers services which improve the biologists working environment. On going work include the testing phase of the system. In the future we intend to investigate the adaptability of the architecture and GUI to different applications.

\section{References}

[1] Minns, C.K. "Quantifying 'No Net Loss' of Productivity of Fish Habitats", Canadian Journal of Fisheries and Aquatic Sciences (Journal canadien des sciences halieutiques et aquatiques), 54, pp. 2463-2473, 1997.

[2] Minns, C. K., "Science for Freshwater Fish Habitat Management in Canada: Current Status and Future Prospects". Aquatic Ecosystem Health and Management, 4, pp. 423-436, 2001.

[3] Graniero, P.A. and Miller, H.S. "A mobile environmental monitoring system with real-time database updates." CRESTech Innovation Network Annual Meeting, Toronto ON, 2002.

[4] OpenRTU, eSolutionsGroup, http://www.openrtu.com/

[5] Pierce, M., Youn, C.-H., Fox, J., "The Gateway Computational Web Portal: Developing Web Services for High Performance Computing", International Conference on Computational Science, 1, pp. 503-512, 2002.

[6] Hp TR3000, http://h18000.www1.hp.com/products/quickspecs/11909_na.PDF

[7] Sharma, J., "Oracle Spatial", An Oracle technical white paper, Oracle Corp., Redwood City, CA, May 2001.

[8] eSpatial Solutions, http://www.espatial.com/

[9] Olson, S., "Ajax on Java", O’Reilly Media, 2006, ISBN: 0596101872

[10] Marinilli, M., Java Deployment Using JNLP and WebStart, September 2001, ISBN: 0672321823

[11] Carswell, J. D., Gardiner K., Bertolotto M., Mandrak N., "Applications of Mobile Computing for Fish Species at Risk Management" Proceedings of International Conference on Environmental Informatics of International Society of Environmental Information Sciences (ISEIS2004), Regina, Canada, 2004.

[12] Gardiner, K., Rizzini, A., Carswell, J., Bertolotto, M. "MEMS: Mobile Environmental Management System", GIS Research UK, 13th Annual Conference, University of Glasgow, 6th-8th April 2005. 